\title{
Revalorización de métodos ancestrales de tinturado natural en las provincias de Loja y Azuay del sur de Ecuador
}

\section{Assessment of ancient techniques of natural dyeing in the provinces of Loja and Azuay in the south of Ecuador}

\author{
Cecilia Palacios-Ochoa ${ }^{1 *}$, Narcisa Ullauri ${ }^{2}$ \\ ${ }^{1}$ Universidad del Azuay, Facultad de Diseño Arquitectura y Arte, Carrera de Diseño Textil e Indumentaria. Cuenca-Ecuador. \\ ${ }^{2}$ Universidad del Azuay, Facultad de Filosofía Letras y Ciencias de la Educación, Carrera de Turismo. Cuenca-Ecuador.
}

凶Autor para correspondencia: cpalacios@uazuay.edu.ec

\begin{abstract}
Resumen
El objetivo de este estudio fue revalorizar las técnicas ancestrales de tinturado natural y contribuir a la viabilidad de este importante patrimonio cultural inmaterial. Para ello se identificaron los lugares en donde se las practica actualmente. Se recuperaron las técnicas y optimizaron en función de factores como temperatura, tiempo, $\mathrm{pH}$, clase de mordiente y cantidad de vegetal. Se realizaron 21 tratamientos basados en la metodología empleada por los artesanos y 50 tratamientos con variación de los factores más influyentes. Los tintes se aplica-ron a la fibra de alpaca y lana de oveja. Se obtuvieron 71 tonalidades que van desde el amarillo intenso pasando por los tonos rosa, carmesí, anaranjado hasta el marrón oscuro. Se calificaron como tonos interesantes, por la intensidad aparente que presentaron, a los obtenidos de la aplicación del colorante extraído de Brachyotum confertum, Junglas neotrópica y Dactylopius coccus. Las pruebas de control de solidez del color a la luz, al lavado manual y al frote demostraron que el tinturado con la mayoría de especies utilizadas presentó resultados satisfactorios según la valoración de la escala de grises para medir el cambio de color.
\end{abstract}

Palabras clave: revalorización cultural, tinturado natural, método artesanal, fabricación artesanal.

\begin{abstract}
The aim of this study was to assess the ancient techniques of natural dyeing to contribute to the viability of this important intangible cultural heritage. The locations where natural dyeing is still currently practiced were identified. The original techniques were used and optimized based on factors such as temperature, time, $\mathrm{pH}$, mordant class and amount of plant material. 21 treatments were performed based on the methodology of the artisans and 50 treatments with variations of the most influential factors. The dyes were applied to alpaca fiber and sheep wool. 71 shades were obtained, ranging from intense yellow to pink, crimson, orange, and dark brown. The colors obtained from the application of the dye extracted from Brachyotum confertum, Junglans neotropica and Dactylopius coccus were classified as interesting tones due to the apparent intensity they pre-sented. Control tests such as the strength of color in light, manual washing and rubbing showed that the dyeing with most of the species used had satisfactory results according to the grayscale assessment to measure color change.
\end{abstract}

Keywords: cultural-revalorization, natural dyeing, artisan techniques, handmade production. 


\section{Introducción}

Las técnicas de tinturado indígena han sido utilizadas en el Ecuador desde el periodo Formativo (3500 a.C.). En la época colonial, los tintes se convirtieron en mercancías muy valoradas por los españoles debido a la variedad e intensidad de colores que les resultaban atractivos en las mantas de lana que se exportaban al 'viejo mundo' en ese entonces y a los fuertes tributos y demanda europea, principalmente del carmín extraído de la cochinilla (Guirola, 2010; Jaramillo, 1988). En las provincias del sur del Ecuador, Loja y Azuay, existe una considerable producción de textiles artesanales en los que se usan tinturas artificiales y en mínima proporción las naturales. Es importante conocer el estado actual del uso de los colorantes naturales en estas dos regiones, con la finalidad de promover y recuperar el uso tradicional de los mismos. Además de la trascendencia, el rescate de las técnicas ancestrales de tinturado puede servir para dinamizar la economía de las comunidades y contribuir con una forma práctica de tinturado alternativo al uso de los colorantes sintéticos.

El empleo de los colorantes naturales fue desplazado en todo el mundo a raíz de la introducción del primer colorante sintético en 1856 por el químico británico W.H Perkin, pionero en la elaboración de anilinas y el responsable de establecer las bases de una nueva industria química; sin embargo, los tintes naturales no han dejado de ser evidencia de cultura y tradición. En los últimos años ha aumentado la demanda por los productos naturales en vista de la concientización de las personas por la protección del ambiente, es por ello que los tintes naturales representan una importante opción para teñir fibras. Se ha señalado también que los colorantes naturales tienen efectos benéficos para la salud de las personas puesto que algunos de éstos cuando forman parte de la planta tienen la función de absorber los rayos UV y esta función se mantiene en el ámbito textil, además no provocan irritaciones ni alergias. Existen diversas corrientes en los países de Latinoamérica orientadas a revitalizar la técnica de teñido artesanal; así también, los movimientos ecologistas destacan las características no contaminantes de esta actividad como es el uso de colorantes que por su origen son biodegradables y para fijar el tinte no se utilizan sustancias químicas como el cromo o estaño, por lo que no generan residuos tóxicos al ambiente.
Algunos países como Perú, Bolivia y Argentina poseen escritos que recopilan las metodologías ancestrales del teñido natural (Mattenet et al., 2016; Stramigioli, 1991; Pazos, 2017; Marrone, 2010; Guirola, 2010). En el caso de las provincias de Azuay y Loja no existen reportes actuales sobre el rescate o valorización de los conocimientos tradicionales sobre el teñido natural; se dispone de trabajos experimentales aislados sobre algunas clases de tintes.

En este contexto, el objetivo de la presente investigación fue rescatar los saberes ancestrales relacionados con el uso de las plantas nativas para el teñido artesanal en las provincias de Loja y Azuay, ubicadas al sur del Ecuador.

La estandarización de los procesos permitió mejorar la calidad del tinturado. Con su aplicación se podrá obtener un valor agregado en los productos textiles artesanales, otorgando además a las comunidades el poder de diferenciarse. También se recopiló información sobre el estado de conservación de los vegetales para que se pueda hacer un uso sostenible de dichos recursos.

\section{Materiales y métodos}

Se desarrollaron actividades que incluyeron la recuperación, recreación y optimización de las técnicas de tinturado natural y permitieron combinar los conocimientos ancestrales con los fundamentos de la ciencia para un aprovechamiento sustentable por lo que fue de suma importancia la participación de los artesanos de la región de Namarín y Gualaceo, quienes realizaron las demostraciones de las técnicas que aplican para tinturar. Estas técnicas van transmitiéndose de padres a hijos de forma oral.

\subsection{Determinación de la situación actual del tintu- rado natural}

Se aplicaron 30 encuestas entre los artesanos del área textil de las provincias de Loja y Azuay, lo que sirvió para establecer los principales problemas que afrontan los artesanos e identificar los lugares en donde se practica el tinturado natural. Se realizaron visitas a los centros de Namarín, y Gualaceo, que son los dos sitios principales de tinturado natural, complementando la información con nueve 
entrevistas a adultos mayores dedicados a esta actividad. Las entrevistas proporcionaron datos sobre las metodologías tradicionales que se aplican en las dos regiones y los tipos de plantas que se utilizan comúnmente para el teñido.

\subsection{Recolección y preparación del material}

Se recolectaron las plantas tintóreas en una cantidad que varió de 1 a $2 \mathrm{~kg}$ en las localidades de Namarín, Gualaceo y en los alrededores del cantón Cuenca. Se aplicaron algunas normas como cortar no más del $20 \%$ del vegetal de su parte inferior para no afectar su crecimiento. Se utilizaron herramientas de corte desinfectadas para evitar la propagación de plagas como hongos y bacterias.

Las especies vegetales utilizadas fueron seis que corresponden a los nombres comunes de Barba de viejo, Nogal, Aliso, Killuyuyo, Garau y Shirán. Además, se utilizó cochinilla colorante de origen animal. Se consiguió el que se emplea en la industria alimentaria de origen peruano, y que es usado por algunos artesanos. En nuestro medio la cochinilla se produce en pequeñas cantidades, de forma espontánea y esporádica en Susudel, parroquia rural situada al sur de la provincia del Azuay.

Inmediatamente después de la recolección se realizó la identificación taxonómica con la ayuda de los investigadores del "Herbario Azuay" perteneciente a la Universidad del Azuay, se recabó información sobre el estado de conservación de las diferentes plantas con la finalidad de disponer de datos que permitan hacer un uso sustentable de estos recursos tintóreos. Se estableció que la mayoría se encontraban en un estado de conservación de preocupación menor, si bien algunos son endémicos de esta zona como el Brachyotum confertum, otros son silvestres y algunos de cultivo de rotación.

Se cortaron y separaron las hojas, tallos y cortezas y se procedió a realizar el secado de los vegetales que no se utilizaron de forma inmediata a una temperatura de $40{ }^{\circ} \mathrm{C}$ y se almacenaron empaquetados en papel kraft. Las partes más duras, como tallos y cortezas, se trituraron o molieron y las hojas y flores se cortaron en pedazos pequeños para permitir una mejor extracción del tinte. Algunas plantas necesitaron una fermentación de aproximadamente una semana, lo que se hizo en medio acuoso con las partes de la planta cortadas en pequeños pedazos colocados en recipientes de barro y a temperatura ambiente.

\subsection{Extracción de los colorantes}

La extracción se realizó sometiendo el material vegetal a ebullición en agua durante 1 hora, en un recipiente de acero inoxidable. Se utilizaron 100 $\mathrm{g}$ de vegetal para un volumen de 1 litro de agua, también se ensayó con 200 g de vegetal con lo que se intensificaban los tonos obtenidos. Después de la ebullición se filtra la solución a través de un colador y en caso necesario se ayuda de gasa para retener los residuos vegetales.

\subsection{Preparación de las fibras para el tinturado}

Se utilizaron fibras de alpaca y lana de oveja, hiladas manualmente e industrialmente. Las fibras se lavaron inicialmente en agua caliente y luego en solución de jabón neutro al $0,1 \%$. Se secaron a la sombra antes de tinturarlas. Se utilizaron mordientes que son sustancias químicas que sirven para fijar el color a las fibras de manera uniforme y estable al contacto de la luz y el agua. Se ensayó un premordentado que consiste en colocar las fibras en la solución del mordiente media hora antes de colocar la fibra en el baño del tinte, esto no presentó diferencia con los resultados que se obtuvieron al realizar el mordentado directo, esto es, colocando la solución de mordiente junto con la solución del tinte al mismo tiempo.

\subsection{Ensayos de recreación y optimización de técnicas}

Se realizaron 21 tratamientos con la metodología tradicional de los artesanos rescatada mediante las entrevistas. El método consistió en la utilización del extracto del pigmento mediante ebullición del material vegetal durante una hora y la aplicación directa de la fibra a la solución de la tintura durante media hora con la adición de sal como mordiente en cantidad indeterminada. Con base en la metodología de los artesanos se realizaron otros tratamientos teniendo como variables independientes el $\mathrm{pH}$, tipo de mordiente, temperatura y tiempo de aplicación de la tintura. Se determinó que mejores resultados se obtienen al utilizar de 100 a $200 \mathrm{~g}$ de vegetal para una extracción de 1 hora a ebullición, en una cantidad de $1000 \mathrm{ml}$ de agua. 
Se trabajó con muestras de $10 \pm 2$ g de fibra textil de alpaca y lana de oveja, con una relación de baño de 1/100 con agitación constante. La aplicación del pigmento extraído se realizó durante media y una hora a temperaturas de $70^{\circ} \mathrm{C}, 80^{\circ} \mathrm{C}$ y a temperatura de ebullición. A la temperatura de ebullición se produjo alteración de la fibra de alpaca, por lo que se determinó que la temperatura más adecuada es de 80 grados y no existió mayor diferencia al aplicar el tinte por media o una hora, por lo que se optó por la aplicación de media hora.

Se probaron los mordientes sal y alumbre, en las concentraciones de 1, 2 y $3 \mathrm{~g} / \mathrm{L}$. El mordiente que dio mejores resultados aparentes fue el alumbre y las concentraciones utilizadas no presentaron diferencias aparentes por lo que se adoptó para la aplicación la concentración de alumbre de $1 \mathrm{~g} / 10 \mathrm{~g}$ de fibra. Se utilizó también crémor tártaro y sal marina, los cuales se eligieron teniendo en cuenta la toxicidad y las de uso en la industria alimentaria. Se determinó la menor cantidad necesaria para la fijación. La influencia del $\mathrm{pH}$ en el poder del tinturado se determinó utilizando cantidades medidas de 10, 15 y $20 \mathrm{cc}$ de jugo de limón y midiendo el $\mathrm{pH}$ de la tintura. Al variar el $\mathrm{pH}$ a valores bajos (ácidos) se obtuvieron tonos más débiles. Para la fibra de alpaca que presentó mayor resistencia al teñido, se ensayaron otros mordientes y también para obtener otras tonalidades con la cochinilla se utilizaron sulfato de cobalto (ii), cloruro férrico, sulfato de hierro (II). Después de la aplicación del tinte, se lavó con agua y se secaron las muestras a la sombra.

\subsection{Pruebas de control de calidad}

- Solidez del color a luz. Se utilizó el equipo Lumitester que funciona con una lámpara de mercurio halogenado de electrodos de tungsteno y vapor de mercurio a $400 \mathrm{w}$. La exposición a esta lámpara se correlaciona muy bien tanto con el arco de xenón como con la luz del día. Para esta prueba se prepararon los hilos teñidos enrollándolos a la mascarilla de prueba dejando una parte cubierta y una descubierta para evaluar el cambio de color. La exposición se hizo durante dos horas luego de lo cual se guardaron las muestras en obscuridad a temperatura ambiente y por un lapso de 4 horas. Finalmente, se comparó la porción expuesta con la parte no expuesta de la muestra y se cuantificó el cambio de color usando la escala de grises AATCC (American Association of Textile Chemists and Colorists) para cambio de color. La escala al poseer pares de muestras de grises con diferencias progresivas de los tonos grises permite evaluar visualmente el cambio de color debido a las pruebas a las que se somete la muestra teñida. La nomenclatura de evaluación de las pruebas fue: satisfactorio de $0-10 \%$ de cambio, que corresponde a la valoración de 5 de la escala de grises; y, no admisible para un cambio mayor al 20\% que corresponde a la valoración 1-2 de la escala de grises (Mejía-Azcarate, 2015; Gálvez, 1999).

- Prueba de transferencia del color al frote. Esta prueba se practicó en húmedo y seco, para lo cual se utilizaron telas estándar con las que se hizo un frote manual por veinte veces, tanto mojando la tela con agua destilada como con la tela seca, comparando la tela con la escala cromática AATCC de transferencia de color para evaluar la prueba (Alonso, 2015).

- Prueba de cambio del color al lavado. Esta prueba consistió en producir un lavado que corresponda a cinco lavados domésticos utilizando dos tipos de jabón uno líquido de $\mathrm{pH}$ neutro $\mathrm{y}$ un detergente sólido de uso común de $\mathrm{pH}$ alcalino en una concentración de $5 \mathrm{~g}$ de jabón en $1 \mathrm{~L}$ de agua destilada (Alonso, 2015). Se realizaron pruebas a temperatura de $40{ }^{\circ} \mathrm{C}$ y en agua fría, la prueba en agua caliente provocó una pérdida importante de color por lo que se optó por la prueba en agua fría por un tiempo de 30 minutos con agitación manual continua, se aclaró con agua corriente fría del grifo y se secó a temperatura ambiente. Se evaluó el cambio de color con la escala de grises.

\section{Resultados y discusión}

Las encuestas aplicadas a los artesanos del sector textil mostraron que la totalidad de ellos conoce la importancia de preservar la cultura y los saberes ancestrales y considera que no existe interés de las nuevas generaciones por estos conocimientos. Las principales dificultades que afrontan son la falta de 
apoyo gubernamental, difusión y asesoramiento técnico (Figuras 1 y 2). Además, se determinó que los principales centros donde se practica hasta hoy el tinturado natural están en la comunidad de Ñamarín, cerca de Saraguro, cantón de la provincia de Loja, y en el cantón Gualaceo de la provincia del Azuay.

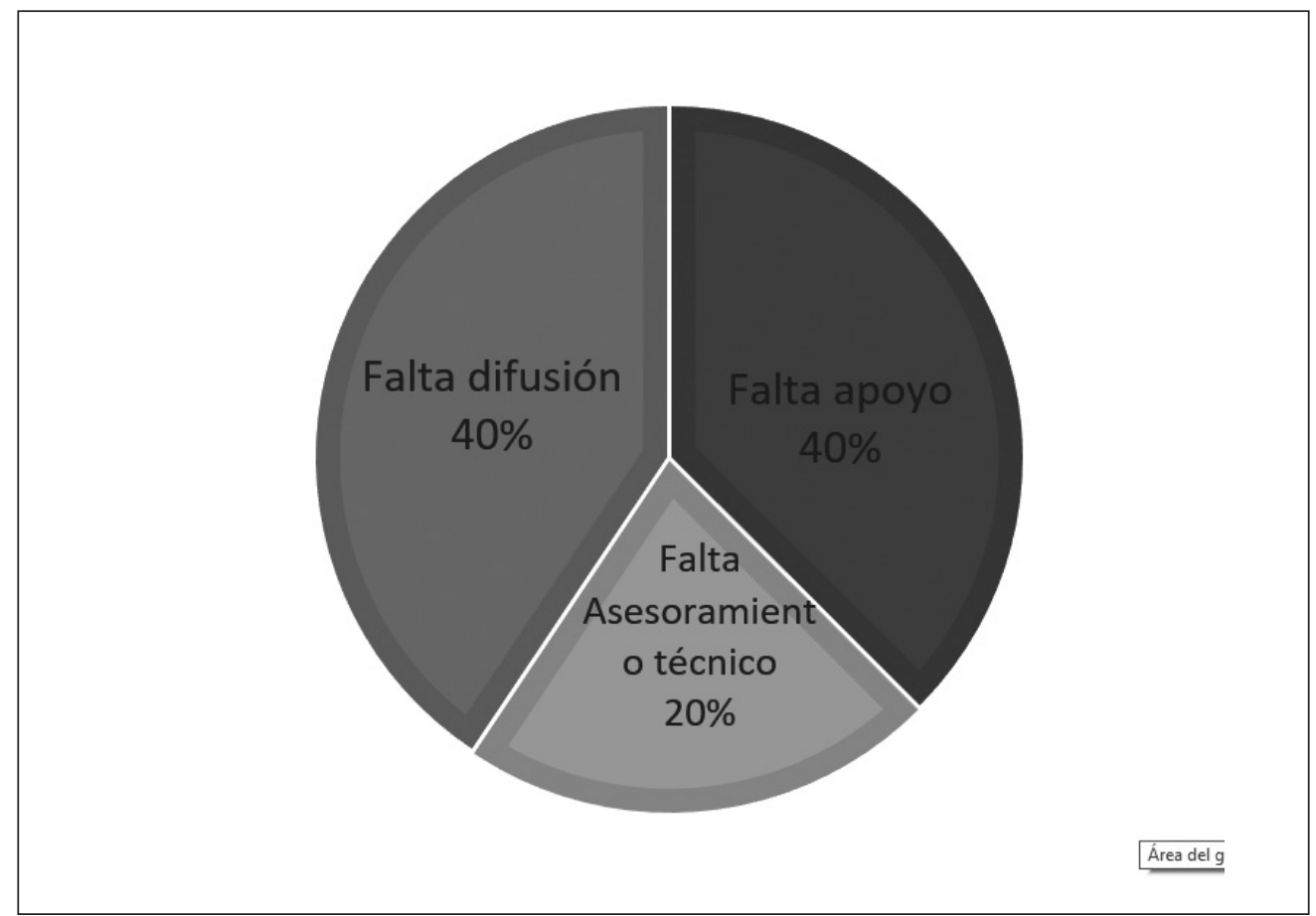

Figura 1. Principales problemas que afrontan los artesanos en la provincia de Loja.

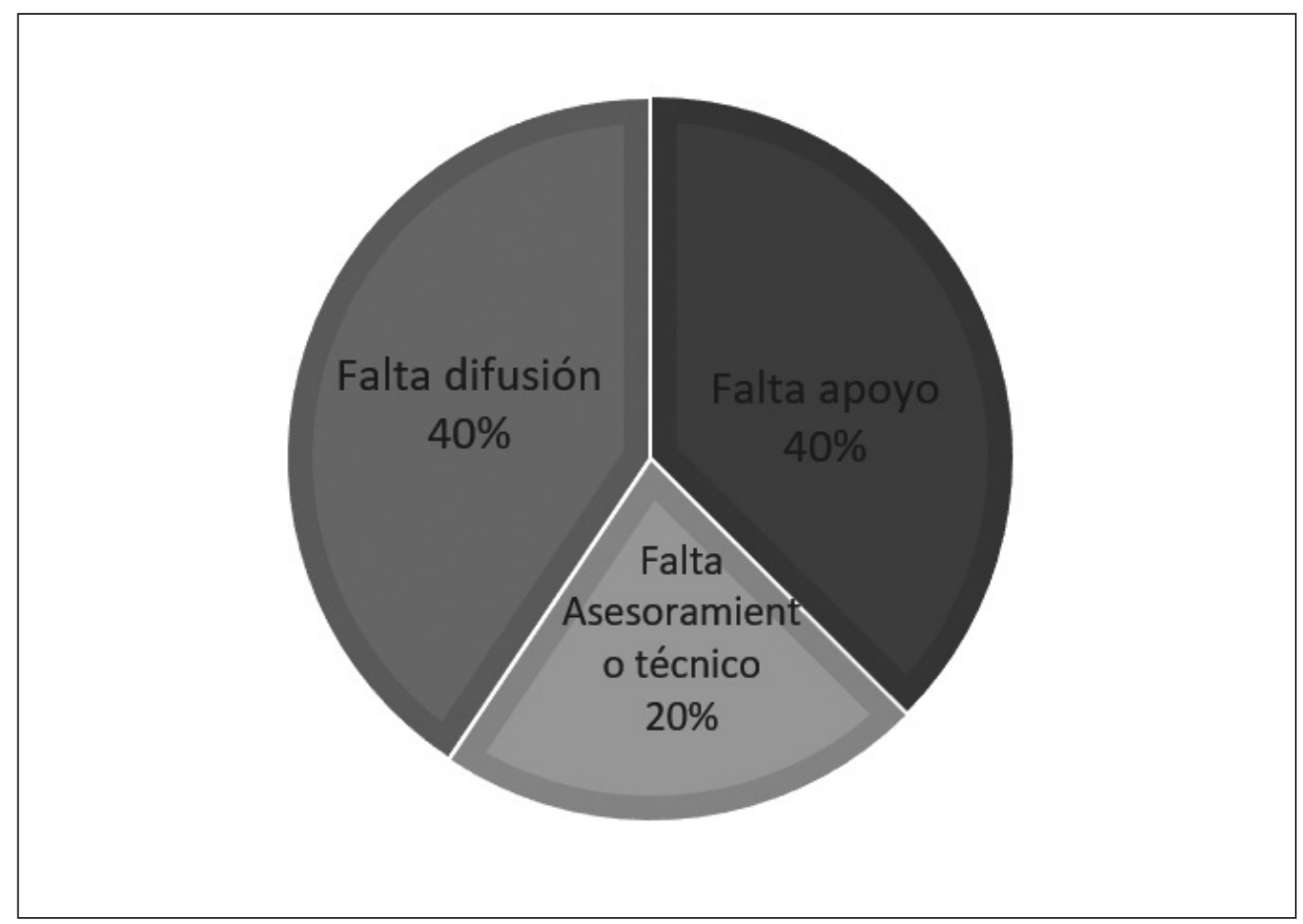

Figura 2. Principales problemas que afrontan los artesanos en la provincia de Azuay. 
Los principales recursos naturales utilizados para el tinturado son las especies de plantas y la de origen animal que se detallan en la Tabla 1. Especies similares de plantas han sido utilizadas desde épocas anteriores en varios países latinoamericanos y en México para teñir fibras naturales, como se reporta en algunos tratados sobre el rescate del tinturado natural (Mattenet et al., 2016; Stramigioli, 1991; Pazos, 2017; Marrone, 2010).

Tabla 1. Especies vegetales y animales comúnmente utilizadas para la extracción de colorantes en las provincias de Loja y Azuay

Azuay

\section{Garau- Lomatia irsuta}

Nogal- Juglans neotrópica

Aliso- Alnus glutinosa

Killuyuyo- Brachyotum confertum

Laurel- Cordiaalliodora

Romero- Rosmarinus officinalis

Chilca- Rosmarinus officinalis
Loja

Fuente: Elaboración a partir de los datos proporcionados por el Herbario Azuay de la Universidad del Azuay.

Tabla 2. Principales colores obtenidos sobre la lana de oveja y codificados con pantone mediante inteligencia artificial y métodos K-means.
Código de
muestra
Especie vegetal o animal
Color
Código Pantone

002

Nogal

004

006

007

008

010

014
Killuyuyo
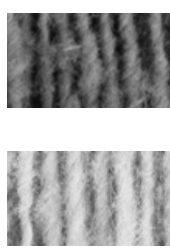

“Sandstorm”,[236,213,64]

“Pantone 611 C”,[215,200,38]

Killuyuyo
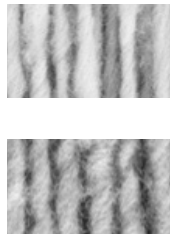

"Pantone 608 C",[233,225,134]

“Pantone 610 C",[225,213,85]

“Vegas gold”,[197,179,88] 


\begin{tabular}{|c|c|c|}
\hline 015 & Nogal & “Otter brown”,[101,67,33] \\
\hline 019 & Barba viejo & "Yellow Sand FS 20266",[162,135,68] \\
\hline 023 & Shirán & “Pantone 459 C”,[222,205,99] \\
\hline 027 & Nogal & "Pantone 7504 C",[148,121,93] \\
\hline 028 & Cochinilla & "Pantone $682 \mathrm{C} "$ \\
\hline 030 & Cochinilla & “Crayola Copper”,[218,138,103] \\
\hline 033 & Cochinilla & "Pantone 687 C",[190,132,163] \\
\hline
\end{tabular}

Fuente: Fotografías de Sebastián Padrón; Laboratorio de Tinturado Universidad del Azuay, 2017

Tabla 3. Principales colores obtenidos sobre la fibra de alpaca y codificados con pantone mediante inteligencia artificial y métodos K-means.

\begin{tabular}{|c|c|c|c|}
\hline $\begin{array}{l}\text { Código de } \\
\text { muestra }\end{array}$ & Especie vegetal o animal & Color & Código Pantone \\
\hline 102 & Aliso & & “Sand FS 33690”,[227,207,183] \\
\hline 103 & Aliso & & “Pantone 7501 C",[217,200,158] \\
\hline 112 & Cochinilla & & "Pantone $7531 \mathrm{C} ",[122,104,85]$ \\
\hline 113 & Cochinilla & & "Pantone 473 C",[240,191,155] \\
\hline 115 & Cochinilla & & “Pantone 720 C",,[231,183,138] \\
\hline 121 & Garau & & “Dark Yellow FS 33448”,[165,146,114] \\
\hline
\end{tabular}




\begin{tabular}{|c|c|c|}
\hline 124 & Killuyuyo & “Pantone 7499 C”,[241,230,178] \\
\hline 126 & Killuyuyo & "Pantone $601 \mathrm{C} ",[240,233,145]$ \\
\hline 127 & Killuyuyo & “Pantone 460 C",[228,215,126] \\
\hline 132 & Nogal & "Night Tan FS20450",[171,150,123] \\
\hline 133 & Killuyuyo & “Pantone 458 C”,[217,199,86] \\
\hline 135 & Cochinilla & “Pantone 687 C",[190,132,163] \\
\hline 136 & Cochinilla & “Pink3”,[205,145,158] \\
\hline
\end{tabular}

Fuente: Fotografías de Sebastián Padrón; Laboratorio de Tinturado Universidad del Azuay, 2017

Mediante las visitas técnicas y entrevistas a los artesanos de las localidades de Namarín y Gualaceo, se propició la participación de los artesanos y se recuperaron las metodologías tradicionales del tinturado. Se realizó la recreación y optimización de las técnicas en el laboratorio. Los mejores resultados en apariencia e intensidad de color se obtuvieron al someter los vegetales a trituración o molido y al utilizar de 100 a 200 g para una cantidad de $10 \mathrm{~g}$ de fibra textil, en una relación de baño de 1/100. La temperatura adecuada para la aplicación de la tintura fue la de $80^{\circ} \mathrm{C}$ por 30 minutos con la que no se observó alteración de la fibra.

Se obtuvieron 71 tonalidades, se calificaron como tonalidades interesantes por la intensidad aparente que presentaron a los obtenidos de $\mathrm{Bra}$ chyotum confertum, Dactylopius coccus, y Juglans neotropica, con la aplicación del mordiente alumbre. Se obtuvieron tonalidades menos intensas al disminuir el $\mathrm{pH}$, esto es debido a que el $\mathrm{pH}$ tiene efecto en la estructura y estabilidad de los pigmentos (Garzón, 2008). El mismo efecto se obtiene cuando se utiliza menores cantidades de vegetal o en la segunda aplicación de la tintura, esto se da por el agotamiento del colorante en el baño. Resultados similares se reportan en el documento escrito sobre conocimientos y técnicas ancestrales de artistas textiles de Perú y Bolivia en el que se recopilan los resultados experimentales de un encuentro realizado con las artesanas de los dos países (Pazos, 2017).

Los colores obtenidos al aplicar la metodología artesanal sin mordiente o con sal presentó colores opacos y sin brillo, debido a que los mordientes como el alumbre tienen la capacidad de fijar de mejor manera el colorante por la reacción con la estructura química de la fibra y el colorante, dotándole al textil de luminosidad (Alonso, 2015). Los resultados sobre los tonos de mayor intensidad y resistencia obtenidos y codificados con pantone se pueden observar en las Tablas 2 y 3. 
Las pruebas de solidez del color, practicadas sobre el tinturado obtenido con la mayoría de especies mostraron resultados satisfactorios con una valoración de 4-5 o 5, al realizar la valoración me- diante la escala de grises AATCC para transferencia y cambio de color, no así el tinturado con aliso que mostró un resultado cuya valoración de 2-3 no es aceptable (Tabla 4).

Tabla 4. Resultados de pruebas de calidad y su valoración según la escala de grises para transferencia y cambio de color

\begin{tabular}{|c|c|c|c|c|c|c|c|c|c|}
\hline \multirow{2}{*}{$\begin{array}{l}\text { Código de } \\
\text { muestra }\end{array}$} & \multirow{2}{*}{$\begin{array}{c}\text { Número } \\
\text { de } \\
\text { muestra }\end{array}$} & \multirow{2}{*}{ Tinte } & \multirow{2}{*}{ Tono } & \multirow{2}{*}{ Base textil } & \multicolumn{2}{|c|}{ Lavado } & \multicolumn{2}{|c|}{ Frote } & \multirow{2}{*}{$\begin{array}{c}\begin{array}{c}\text { Solidez a } \\
\text { la luz }\end{array} \\
2 \text { horas }\end{array}$} \\
\hline & & & & & $\begin{array}{l}\text { Jabón } \\
\text { neutro }\end{array}$ & $\begin{array}{c}\text { Jabón } \\
\text { alcalino }\end{array}$ & Húmedo & Seco & \\
\hline 033 & 1 & Cochinilla & Fucsia & Lana & $4-5$ & $2-3$ & 5 & 5 & 5 \\
\hline 015 & 2 & Nogal & Marrón & Lana & 5 & 5 & 5 & 5 & 5 \\
\hline 006 & 3 & Killuyuyo & Amarillo & Lana & 5 & 5 & 5 & 5 & 5 \\
\hline 116 & 4 & Cochinilla & Naranja & Lana & $4-5$ & 3 & 5 & 5 & 5 \\
\hline 115 & 5 & Cochinilla & Rosa & Alpaca & 4 & $2-3$ & 5 & 5 & 5 \\
\hline 010 & 6 & Garau & Marrón & Lana & $4-5$ & $3-4$ & 4 & 5 & 5 \\
\hline 031 & 7 & Cochinilla & Naranja & Alpaca & 4 & $2-3$ & 5 & 5 & 5 \\
\hline 116 & 8 & Cochinilla & Rosa & Baby alpaca & 4 & $2-3$ & 5 & 5 & $4-5$ \\
\hline 019 & 9 & Barba de viejo & Marrón & Lana & $4-5$ & 3 & $4-5$ & 5 & 5 \\
\hline 023 & 10 & Shirán & Amarillo & Lana & 5 & 5 & 5 & 5 & 5 \\
\hline 014 & 11 & Aliso & Marrón & Lana & $2-3$ & 2 & 2 & 3 & $2-3$ \\
\hline 133 & 12 & Killuyuyo & Amarillo & Baby alpaca & 5 & $2-3$ & 5 & 5 & 5 \\
\hline 106 & 13 & Aliso & Marrón & Alpaca & $2-3$ & 2 & 2 & 3 & $2-3$ \\
\hline 137 & 14 & Barba de viejo & Amarillento & Baby alpaca & 5 & $3-4$ & 5 & 5 & $4-5$ \\
\hline 121 & 15 & Garau & Marrón & Alpaca & $4-5$ & $3-4$ & $3-4$ & 5 & $4-5$ \\
\hline 131 & 16 & Nogal & Marrón & Alpaca & 5 & 5 & 5 & 5 & 5 \\
\hline
\end{tabular}


Las pruebas de calidad demostraron que el tinturado con la mayoría de vegetales ensayados tuvo la calificación de satisfactorio, lo que sirvió para demostrar que las técnicas ancestrales de teñido se pueden aplicar de una manera tecnificada para obtener mejores resultados.

\section{Conclusiones}

Los resultados de la indagación muestran que las técnicas ancestrales de tinturado natural se encuentran en peligro de desaparecer y las experimentaciones a nivel de laboratorio demostraron que con la optimización de los métodos tradicionales se mejoró la calidad del tinturado en cuanto a la intensidad aparente y a la solidez de los colores. El tinturado presentó mayor intensidad y brillo con el uso del mordiente alumbre, mientras que la variación del $\mathrm{pH}$ produjo una modificación del color a tonos más dé-

\section{Referencias}

Alonso, F. (2015). Manual de control de calidad en productos textiles y afines. Madrid: Universidad Politécnica de Madrid.

Gálvez, F. (1999). Un aporte más al diseño industrial. Normatividad en textiles para tapicería de mobiliario. México: Universidad Nacional Autónoma de México

Garzón, G. (2008). Las antocianinas como colorantes naturales y compuestos bioactivos: Revisión. Acta Biológica Colombiana, 13(3), 27-36

Guirola, C. (2010). Tintes naturales y su uso en Mesoamérica desde la época prehispánica. Guatemala: Asociación Flaar Mesoamérica.

Jaramillo, H. (1988). Textiles y tintes (1a ed.). Cuenca: Centro Interamericano de Artesanías y Artes Populares (CIDAP).

Marrone, L. (2010). Tintes naturales al alcance de nuestras manos. Guía práctica para el teñido de lanas con colorantes naturales. Argentina: Editorial Martin. biles. La aplicación de los colorantes extraídos de la mayoría de las especies vegetales principalmente de Branchyotum confertum (killuyuyo), Juglans neotrópica (nogal) y de la especie animal Dactylopius coccus (cochinilla) presentaron tinturados con una solidez al lavado, frote y exposición a la luz, con una valoración correspondiente a resultados satisfactorios según la escala de grises para la transferencia y cambio de color, no así el obtenido con la especie Alnus glutinosa (aliso) que presentó cambios de color inaceptables.

La estandarización de los procesos permitirá la reproducción de los colores y obtención de productos textiles artesanales de mejor calidad. Con la posterior transmisión de las técnicas ancestrales validadas se contribuirá a la salvaguardia de este patrimonio y se promoverá además la protección del ambiente y la salud de la comunidad al utilizar productos naturales.

Mattenet, F., Goyheneix, M., \& Peri, P. (2016). Tintes naturales de plantas nativas: colores de la Patagonia (1a ed.). Santa Cruz: Secretaria de Agricultura Familiar Santa Cruz.

Mejía-Azcarate, F. (2015). Programa de textilización. Una herramienta completa y didáctica para poder entender el complejo mundo de la fabricación textil (6a ed.). Recuperado de: https://programadetextilizacion.blogspot.com/

Pazos, S. (2017). Teñido en base a tintes naturales: Conocimiento y técnicas ancestrales de artistas textiles de Perú y Bolivia (1a ed.). Perú: Practical Action.

Stramigioli, C. (1991). Teñido con colorantes naturales. Recuperación de una técnica tradicional. Argentina: Ediciones Ayllu. 